\title{
Low Intensity Aerobic Exercise Improves the Motor Perceptual Functions in Aged Males with Insomnia
}

\author{
Omid Jamshidi ${ }^{1}$, Ali Seghatoleslami² ${ }^{\star}$, Maryam Salmanpour ${ }^{3}$ \\ ${ }^{1} \mathrm{PH} . \mathrm{D}$ of Sport Sciences, Imam Khomeini International University, Qazvin, Iran \\ ${ }^{2}$ Assistant Professor of sport sciences, University of Birjand \\ ${ }^{3}$ Master of Exercise Physiology, Department of Exercise Physiology, University of Tehran, Tehran, Iran
}

\begin{abstract}
A growing literature supports the detrimental consequences of insomnia on cognitive, motor functioning and psychomotor performance such as motor and cognitive reaction time in aged populations. Thus, the purpose of the research was to investigate the effect of low intensity aerobic on perceptual motor functions in aged males with insomnia. 31 elderly males with insomnia were randomly assigned to either exercise $(n=16)$ or control groups $(n=15)$. Exercise group engaged in 30 min of aerobic exercise 3 times per week for 16 weeks with intensity of 40-50\% of maximal heart rate. Perceptual motor functions and self-reported sleep quality were assessed at baseline and at 16 weeks by the Vienna System Test and Pittsburgh Sleep Quality Index (PSQI) respectively. Independent and dependent t-tests were used to analyze the data. It was suggested that both motor and cognitive reaction time were improved after exercise training $(p=0.001)$, meanwhile no significant change was found in the control group ( $p \geq 0.05$ ). Furthermore, quality of sleep was improved significantly in the exercise group ( $p=0.001)$. Although insomnia could result in perceptual motor functions in aged populations, the aerobic exercise would alleviate the deleterious effect of insomnia in decisive task among elderly. The result raises the possibility that doing the light aerobic exercise may be a key strategy for increasing the potentials of perceptual motor functions of aged population.
\end{abstract}

Keywords: Aged, insomnia, PSQI, exercise

\section{INTRODUCTION}

Medical advancements have yielded the extended life expectancy among elderly worldwide. In this regard, an accelerating rate of aging population by 2032 has been reported in Iran (Amini, Mirmoezzi, Salmanpour, \& Khorshidi, 2018). Promotion plans for successful aging

\footnotetext{
*Correspondence: aseghatoleslami@birjand.ac.ir

Ali Seghatoleslami, Department of Physical Education, Faculty of Physical

Education, University of Birjand, Birjand, Iran
}

Phone:989155623745 Fax:985632202032

Received: 14 April 2018 Accepted: 18 April 2018

Sleep and Hypnosis

Journal homepage:

www.sleepandhypnosis.org

ISSN: 2458-9101 (Online) can improve the physical and mental health and decrease the possible costs of healthcare systems. Bodily changes are common signs of aging which threatens the mental performance in the age groups. Fatigue owing to either physical limitations or any behavioral problem such as insomnia possesses some challenges in cognitive performance of aged people. A number of studies have shown increased levels of mood disturbances, anxiety, fatigue in individuals with insomnia in comparison with normal sleepers, although these symptoms usually remain in the subclinical range. Thus, it's necessary to attenuate the negative consequences of degenerative consequences of aging. Hence, optimal physical and psychological performance during aging period is of utmost importance in the view of health authorities, so 
knowing about the important factors which positively affect personal independency in the dimensions is a topic of great interest among researchers (Irandoust \& Taheri, 2015). A growing literature supports the detrimental consequences of insomnia on human cognitive, motor functioning and psychomotor performance such as reaction time and memory tasks (Mah, Mah, Kezirian, \& Dement, 2011; Patrick et al., 2017; Scott, McNaughton, \& Polman, 2006). On the other hand, the deleterious effect of acute sleep deprivation on endurance tasks has been well documented (Fullagar et al., 2015; Thun, Bjorvatn, Flo, Harris, \& Pallesen, 2015). Other studies have shown that sleep deprivation ranging from 30 to $64 \mathrm{~h}$ influences simple and choice reaction time significantly (Philip et al., 2003). Although numerous studies have investigated the influence of exercise on quality of sleep, few research can be found relating to the role of exercise in mitigating the adverse effects of insomnia (Fortier-Brochu, Beaulieu-Bonneau, Ivers, \& Morin, 2010). Based on scientific evidences, any disturbance factor which adversely affects the sleep cycle, attenuate person's ability to perform a variety of perceptual motor tasks required in daily performance. Of the measures of perceptual motor factors which is mostly influenced by insomnia, cognitive and motor reaction time are the two important parameters playing vital role in information processing (Berg \& Neely, 2006). Owing to the importance of perceptual motor performance in aged persons, it was postulated that light aerobic exercise would affect perceptual motor tasks which plays a mediator role in attenuating adverse effect of insomnia on perceptual motor functions of elderly.

\section{METHODS}

33 elderly males with insomnia were recruited voluntarily in the research, and provided written informed consent. Two of them were removed from the study. They were randomly assigned to either exercise $(n=16)$ or control groups $(n=15)$. All subjects were asked to fill the personal information questionnaire regarding the age, weight, height, their background in sports participation, and the amount of training per week. They were also asked to record their sleep-wake schedules for at least a 3-day period before pretest. Inclusion criteria were $P S Q I>5$; aged $\geq 60$ years old; lack of smoking and medication use, no regular exercise within the past six-month. They were also excluded if any of their first degree relatives had a history of primary sleep disorder or major psychiatric illness. They were instructed to refrain from any stimulants such as consuming caffeinated drinks as well as taking nap and doing exercise before pre- and posttests. Participants' time of going to bed varied between 1000 p.m. and 1100 p.m. The time of waking up varied between 0700 a.m. and 0800 a.m. The food programs were given to all subjects in order to mitigate the possible effects of nutrition on study variables. Exercise group engaged in 30 min of aerobic exercise 3 times per week for 16 weeks with intensity of $40-50 \%$ of maximal heart rate (controlled by Polar S610, Kempele, Finland). The exercise program consisted of warm-up (5 minutes with stretching exercises), 30 minutes of aerobic exercises and cool-down in 10 minutes, respectively (Amini et al., 2018; Roveda et al., 2011). Perceptual motor functions and sleep quality were assessed at baseline and at 16 weeks by Vienna system test and PSQI respectively. All procedures performed in studies involving human participants were in accordance with the ethical standards of the institutional and/or national research committee and with the 1964 Helsinki declaration and its later amendments or comparable ethical standards. The study was approved by the Ethics Committee of Imam Khomeini international university. Normality distribution of variables was tested using Kolmogorov Smirnov test. Independent and dependent t-tests were used to analyze the data.

\section{RESULTS}

General characteristics of subjects can be seen in table 1. As shown, no significant difference was found in anthropometry traits of subjects in baseline phase ( $p \geq 0.05$ ). 
Table 1. General characteristics of subjects (mean \pm SD)

\begin{tabular}{llcc}
\hline Group & Exercise & Control & P-value \\
\hline Age $(\mathrm{yr})$ & $2.5 \pm 63.3$ & $3.2 \pm 65.4$ & 0.07 \\
Height $(\mathrm{cm})$ & $5.4 \pm 168.2$ & $6.1 \pm 167.1$ & 0.55 \\
Weight $(\mathrm{kg})$ & $4.2 \pm 66.2$ & $4.3 \pm 67.2$ & 0.12 \\
BMI $\left(\mathrm{kg} / \mathrm{m}^{2}\right)$ & $1.9 \pm 24.1$ & $3.2 \pm 24.3$ & 0.49 \\
\hline
\end{tabular}

Table 2. The effect of Exercise on motor perceptual Functions in (paired-t-test)

\begin{tabular}{|c|c|c|c|c|c|c|c|}
\hline & \multirow{2}{*}{$\begin{array}{l}\text { Group } \\
\text { Tests }\end{array}$} & \multicolumn{2}{|c|}{ Exercise } & \multirow{2}{*}{$\mathbf{P}$} & \multicolumn{2}{|c|}{ Control } & \multirow{2}{*}{$\mathbf{P}$} \\
\hline & & Pretest & Post test & & Pretest & Post test & \\
\hline \multirow[t]{2}{*}{ PMF } & MCRT (m seconds) & $548.2 \pm 19.7$ & $511.3 \pm 21.3$ & $0.001^{*}$ & $545.3 \pm 24.3$ & $551.25 \pm 22.1$ & 0.29 \\
\hline & MMT (m seconds) & $257.3 \pm 7.6$ & $211.1 \pm 8.4$ & $0.001^{*}$ & $251.87 \pm 11.4$ & $260.1 \pm 13.5$ & 0.98 \\
\hline
\end{tabular}

PMF: Perceptual Motor Functions; MCRT: Median Cognitive Reaction Time; Median Motor Time: MMT.

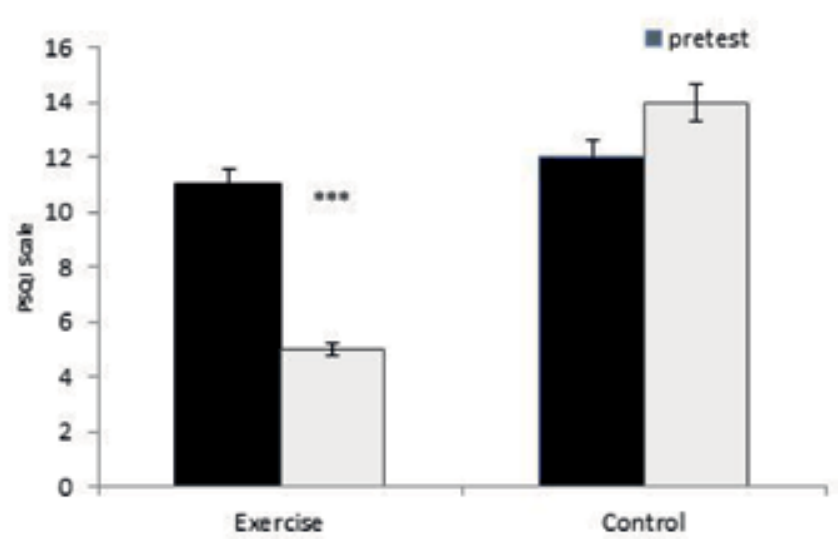

Figure 1. Data are expressed as mean $\pm S D(* * *: p<0.001)$

The independent t-test results suggested that there were no significant differences in baseline tests of sleep quality and perceptual motor performance in both groups $(\mathrm{p} \geq 0.05)$.

As it can be seen in Figure 1, quality of sleep was significantly changed after exercise intervention $(p=0.001$; $t=17.8$ ), while no significant effect was found in control group ( $\mathrm{p} \geq 0.05)$.

The perceptual motor performance of subjects is shown in table 2 . It was suggested that both motor and cognitive reaction time were improved after exercise training ( $p=0.001)$, meanwhile no significant change was found in control group ( $\mathrm{p} \geq 0.05)$.

\section{DISCUSSION}

The present study aimed at assessing the effect of low intensity aerobic exercise on motor perceptual functions in aged males with Insomnia. It was hypothesized that insomnia would show impairments in perceptual motor functions such as motor reaction time and cognitive reaction. The results suggested that both motor reaction time and cognitive reaction time were significantly improved after a light exercise compared to those who didn't have such an exercise intervention. Consistent with our study, some studies supported the deleterious effects of sleep disorder on processing functions (Irandoust \& Taheri, 2018; Monleon, Hemmati Afif, Mahdavi, \& Rezaei, 2018; Taheri \& Arabameri, 2012) suggesting that psychomotor functions such as reaction time, attention and concentration were significantly impaired by nocturnal sleep deprivation, while, the light aerobic exercise would alleviate the deleterious effect of sleep deprivation in decisive task among those who have been in a chronic sleep-restricted state. Besides, it has been shown that exercise therapy could be attributed to an optimal status of sleep quality among elderly (Irandoust \& Taheri, 2018; Taheri \& Irandoust, 2017). It must be noted that the age groups of population studied in these two researches have been variable (aged versus young population). 
Scientific facts support the notion that that the prefrontal cortex is extremely sensitive to sleep disturbance (Fortier-Brochu et al., 2010). A great deal of research has demonstrated the mitigating effect of sleep loss on pre-frontal cortex metabolism which adversely affects the human executive function (Dinges et al., 1997). Furthermore, our results shed some light on the empowering effect of a light aerobic exercise on deleterious effect of insomnia on perceptual motor functions that is consistent with Fullagar study who reportedthat reduction in sleep quality and quantity could result in an autonomic nervous system imbalance, simulating symptoms of the overtraining syndrome. Additionally, increases in pro-inflammatory cytokines following sleep loss could promote immune system dysfunction. Of further concern, numerous studies investigating the effects of sleep loss on cognitive function report slower and less accurate cognitive performance (Fullagar et al., 2015). On the other hand, Divergent results have been reported on the effect of sleep loss on perceptual motor functions (Cain, Silva, Chang, Ronda, \& Duffy, 2011). One reason for getting conflicting findings may be due to the different assessments (i.e., either subjective or objective tools) (Pavlovic et al., 2018; Reza Sharif \& Sayyah, 2018); variable exercise protocols and different age groups of subjects. It must be noted that giving physical health feedback can be of good help for making the people aware of exercise necessity in improvement of health (Irandoust, Taheri, Neto, \& Lotfi, 2017; Rodrigues-Rodrigues, Claudia Vieira Gomes, \& Rodrigues Neto, 2018). Considering the psychological problems such as depression and its interaction with perceptual motor functions is a key issue (Irandoust \& Taheri, 2017) that must be taken into consideration in future studies. In summary, it was shown that nocturnal sleep loss resulting in decrease of motor perceptual functions would be compensated to some extent by light aerobic exercise. These results raise the possibility that doing the light aerobic exercise may be a key strategy for increasing the potentials of perceptual motor functions of aged population. Given the equivocal understanding of sleep and perceptual motor functions, more research involving a more controlled environment with larger sample size is needed to obtain a greater knowledge of the interaction between exercise, sleep and perceptual motor functions.

Acknowledgments: We thank the study participants who generously devote their valuable times for the study.

Conflict of interest: All authors have nothing to declare.

Funding: No funding was received for this research.

\section{References}

Amini, M., Mirmoezzi, M., Salmanpour, M., \& Khorshidi, D. (2018). Eight weeks of aerobic exercises improves the quality of life in healthy aged sedentary men. International journal of Sport Studies for Health, 1(1), e67514. doi:10.5812/intjssh.67514

Berg, J. v. d., \& Neely, G. (2006). Performance on a simple reaction time task while sleep deprived. Perceptual and Motor Skills, 102(2), 589-599. doi:doi:10.2466/pms.102.2.589-599

Dinges, D. F., Pack, F., Williams, K., Gillen, K. A., Powell, J. W., Ott, G. E., .. . Pack, A. I. (1997). Cumulative sleepiness, mood disturbance, and psychomotor vigilance performance decrements during a week of sleep restricted to $4-5$ hours per night. Sleep, 20(4), 267-277.

Fortier-Brochu, É., Beaulieu-Bonneau, S., Ivers, H., \& Morin, C. M. (2010). Relations between sleep, fatigue and health-related quality of life in individuals with insomnia. Journal of Psychosomatic Research, 69(5), 475-483. doi:10.1016/j. jpsychores.2010.05.005

Fullagar, H. H., Skorski, S., Duffield, R., Hammes, D., Coutts, A. J., \& Meyer, T. (2015). Sleep and athletic performance: the effects of sleep loss on exercise performance, and physiological and cognitive responses to exercise. Sports Medicine, 45(2), 161-186.
Irandoust, K., \& Taheri, M. (2015). The effects of aquatic exercise on body composition and nonspecific low back pain in elderly males. Journal of Physical tTherapy Science, 27(2), 433-435.

Irandoust, K., \& Taheri, M. (2017). The effect of vitamin d supplement and indoor vs outdoor physical activity on depression of obese depressed women. Asian Journal of Sports Medicine, $8(3)$.

Irandoust, K., \& Taheri, M. (2018). The effect of strength training on quality of sleep and psychomotor performance in elderly males. Sleep and Hypnosis, 20(3), 160-165.

Irandoust, K., Taheri, M., Neto, G. R., \& Lotfi, L. (2017). Physical and physiological literacy feedback improves the exercise behavior in tofi governors and chief executive officers.

Mah, C. D., Mah, K. E., Kezirian, E. J., \& Dement, W. C. (2011). The effects of sleep extension on the athletic performance of collegiate basketball players. Sleep, 34(7), 943-950.

Monleon, C., Hemmati Afif, A., Mahdavi, S., \& Rezaei, M. (2018). The acute effect of low intensity aerobic exercise on psychomotor performance of athletes with nocturnal sleep deprivation. International journal of Sport Studies for Health, 1(1), e66783. doi:10.5812/intjssh.66783 
Patrick, Y., Lee, A., Raha, O., Pillai, K., Gupta, S., Sethi, S., . . Moss, J. (2017). Effects of sleep deprivation on cognitive and physical performance in university students. Sleep and Biological Rhythms, 15(3), 217-225. doi:10.1007/s41105-017-0099-5

Pavlovic, R., Mihajlovic, I., Idrizovic, K., Vrcic, M., Stankovic, D., \& Joksimovic, M. (2018). Differences in anthropometric traits and trend of changes in high school students. International journal of Sport Studies for Health, 1(1), e68101. doi:10.5812/ intjssh.68101

Philip, P., Sagaspe, P., Taillard, J., Moore, N., Guilleminault, C., Sanchez-Ortuno, M., . . Bioulac, B. (2003). Fatigue, sleep restriction, and performance in automobile drivers: a controlled study in a natural environment. Sleep, 26(3), 277-280.

Reza Sharif, M., \& Sayyah, M. (2018). Assessing physical and demographic conditions of freshman \&quot;15\&quot; male medical students. International journal of Sport Studies for Health, 1(1), e67421. doi:10.5812/intjssh.67421

Rodrigues-Rodrigues, T., Claudia Vieira Gomes, A., \& Rodrigues Neto, G. (2018). Nutritional status and eating habits of professors of health area. International journal of Sport Studies for Health, 1(1), e64335. doi:10.5812/intjssh.64335
Roveda, E., Montaruli, A., Calogiuri, G., Carandente, F., Sciolla, C., $\&$ Angeli, A. (2011). Effects of endurance and strength acute exercise on night sleep quality. International Sport Medicine Journal, 12(3), 113-124.

Scott, J. P., McNaughton, L. R., \& Polman, R. C. (2006). Effects of sleep deprivation and exercise on cognitive, motor performance and mood. Physiology \& Behavior, 87(2), 396408.

Taheri, M., \& Arabameri, E. (2012). The effect of sleep deprivation on choice reaction time and anaerobic power of college student athletes. Asian Journal of Sports Medicine, 3(1), 15.

Taheri, M., \& Irandoust, K. (2018). The exercise-induced weight loss improves self-reported quality of sleep in obese elderly women with sleep disorders. Sleep and Hypnosis, 20(1), 54-59.

Thun, E., Bjorvatn, B., Flo, E., Harris, A., \& Pallesen, S. (2015). Sleep, circadian rhythms, and athletic performance. Sleep Medicine Reviews, 23, 1-9. 